\title{
3D Core-Scale Organic and Mineral Material Characterization of Source Rocks with Simultaneous Neutron and X-Ray Imaging
}

\author{
Weichang $\mathrm{Li}^{1}$, Wei-Shan Chiang ${ }^{2}$, Jacob LaManna ${ }^{3}$, Jordan Kone ${ }^{1}$, Jin-Hong Chen ${ }^{1}$ and Yun Liu ${ }^{2}$ \\ 1. Aramco Research Center - Houston, Aramco Services Company \\ 2. Center for Neutron Research, National Institute of Standards and Technology, Gaithersburg, Maryland \\ 3. Physical Measurement Lab., National Institute of Standards and Technology, Gaithersburg, Maryland
}

Hydrocarbon production from shales based on horizontal drilling and hydraulic fracturing has been the key development for the energy industry in the past decade both in the U.S. and globally. Nevertheless, fundamental questions related to (a) the storage and flow of light hydrocarbons in shales and (b) the geochemical and organofacies characterization of source rocks at the proper scales remain largely unresolved. With respect to (a), hydrocarbons in the shale rocks have been found to be stored primarily within kerogen pores of characteristic scale from 1 to $100 \mathrm{~nm}$. In addition, the 3D connectivity of these kerogen pores and fractures from micron to centimeter form the flow path for light hydrocarbons. Therefore, to better model gas-in-place and permeability in shales, it is necessary to quantify the structural distribution of organic and inorganic components and fractures in shales over a large range of length scales. With respect to (b), although geochemical analysis at microscale such as Fourier transform infrared spectroscopy (FTIR) imaging, confocal fluorescence and Energy-dispersive X-ray spectroscopy (EDX) have been routinely applied to obtain organic and mineral characterization, accurate upscaling of these information to larger dimension at core or log level remains a challenge and an active research topic.

Simultaneous neutron and X-ray tomography [1] offers a core-scale non-destructive method that can distinguish organic kerogen, inorganic minerals, and open and healed fractures in shales with resolution of about $20 \mu \mathrm{m}$ and field of view about $2 \mathrm{~cm}$. In the neutron data, the hydrogen-rich areas, i.e. organic kerogen, are brighter because hydrogen has larger attenuation coefficient and attenuates neutron intensity more significantly [2]. For the X-ray data, the attenuation coefficient of an element is related to its atomic number $Z$ and the brighter areas indicate the region containing more high- $Z$ elements such as minerals [3]. Open fractures do not attenuate either neutron or X-ray and therefore look dark in both neutron and X-ray images.

In this study, shale samples with various maturity and total organic carbon (TOC) were investigated using simultaneous neutron and X-ray tomography. We were able to construct multiple 3D images of shales with different properties such as $3 \mathrm{D}$ kerogen structure, high- $Z$ minerals, and fractures. The constructed 3D maps of kerogen and fractures can be used in the modeling of hydrocarbon flow in core scale, a $10^{9}$ upscaling from current method that models the flow based on scanning electron microscopy (SEM) images. In addition, the core-scale distribution of the organic and mineral materials simultaneously imaged through X-ray and neutron tomography can be tied to geochemical characterization obtained from microanalysis of thin sections taken from the same samples, in an attempt to providing an upscaling basis for maturity and organofacies characterization.

In one example, the X-ray tomography of a source rock sample of 1 inch diameter and 0.75 inch height was taken to produce 1,145 2D image slices, and the neutron image volume consisted of 2,625 slices. These 2D slice images were first filtered to remove noises and enhance features such as fractures and mineral and organic segments. Then the slice stacks for X-ray and neutron images were interpolated along the depth direction to obtain the same sample interval. The filtered and interpolated X-ray and neutron image slices at each depth point were then co-registered to obtain the proper alignment, rotation and scaling, with results from one set of slices given in Figure 1. After these preprocessing steps, the pair-wise registered X-ray and neutron images were segmented, with an example shown in Figure 2, to 
produce the cross-sectional mineral and organic distributions, respectively, at each interpolated depth point. The segmented $\mathrm{X}$-ray slices showing high-Z minerals, and the segmented neutron image slices showing high hydrogen distribution, were then rendered into 3D volumes independently, as shown in Figure 3. Finally, each registered pair of segmented 2D slices from X-ray and neutron images at the same depth point were fused together and rendered in a single volume, producing a 3D representation of both mineral and organic distribution of the core sample, as shown in Figure 4.

As an early attempt to applying simultaneous X-ray and neutron imaging to geological sample characterization, we emphasize that these initial results are still preliminary and further detailed analysis is currently underway such as differentiating water effect from kerogen in neutron images. However, the simultaneous imaging technique does open up an exciting opportunity and potential for joint characterization of organic and mineral distribution at the core scale, with implications relevant but not limited to the applications (a) and (b) mentioned earlier.

References:

[1] LaManna, J., et al, AGU Fall Meeting Abstracts, (2015).

[2] Perfect, E. et al, Earth-Science Reviews 129 (2014) p. 120.

[3] Cnudde, V. and Boone, M.N., Earth-Science Reviews, 123 (2013) p. 1.

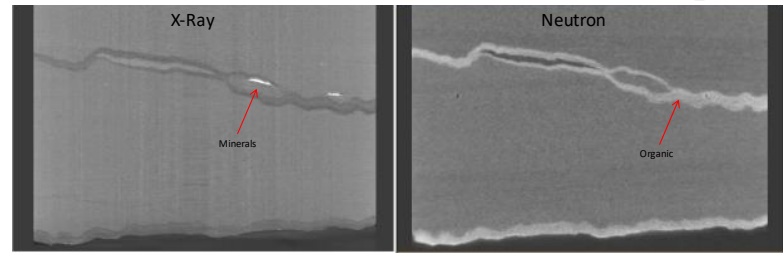

Figure 1. X-ray and neutron image slices, filtered and registered

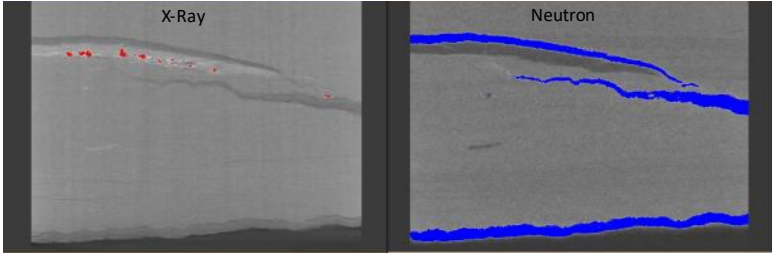

Figure 2. Segmentation of X-ray and neutron image slices to get mineral and organic materials
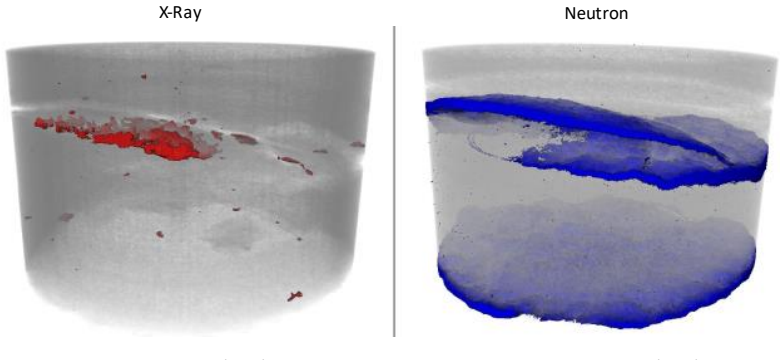

Red $=$ Minerals $.06 \%($ vol $\%)$

Blue $=$ Organic $4.78 \%($ vol $\%)$

Figure 3. Rendered volumes of segmented mineral (X-ray) and organic materials (neutron).

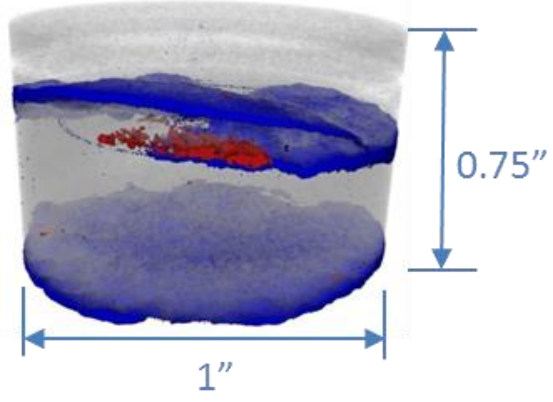

Figure 4. Single rendered volume showing the mineral (red) and organic (blue) material distributions 